
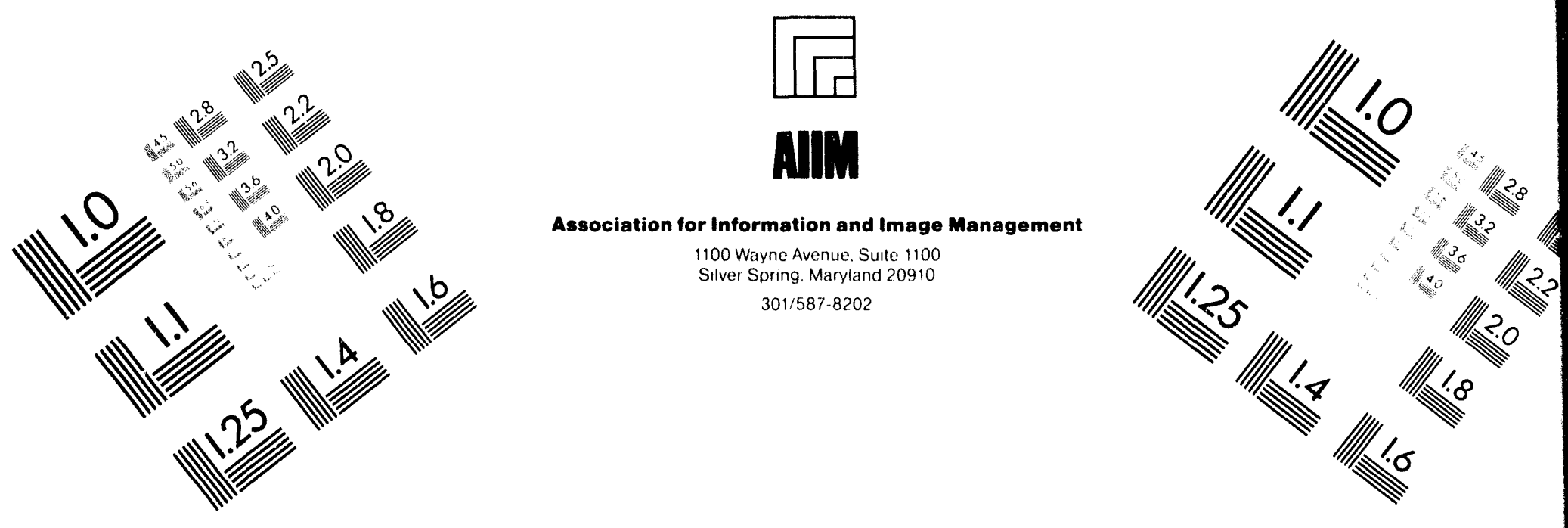

\title{
Centimeter
}

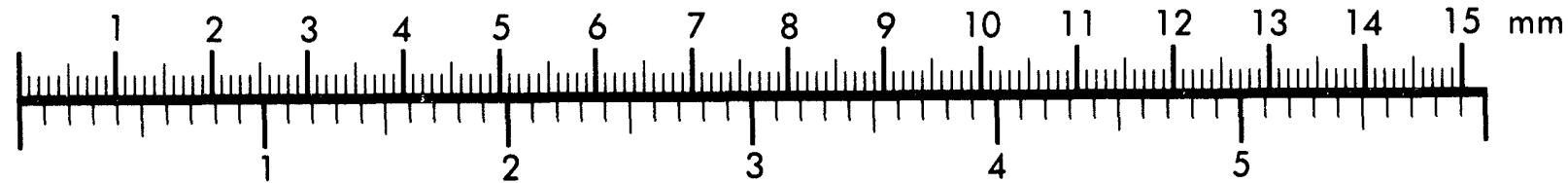
Inches
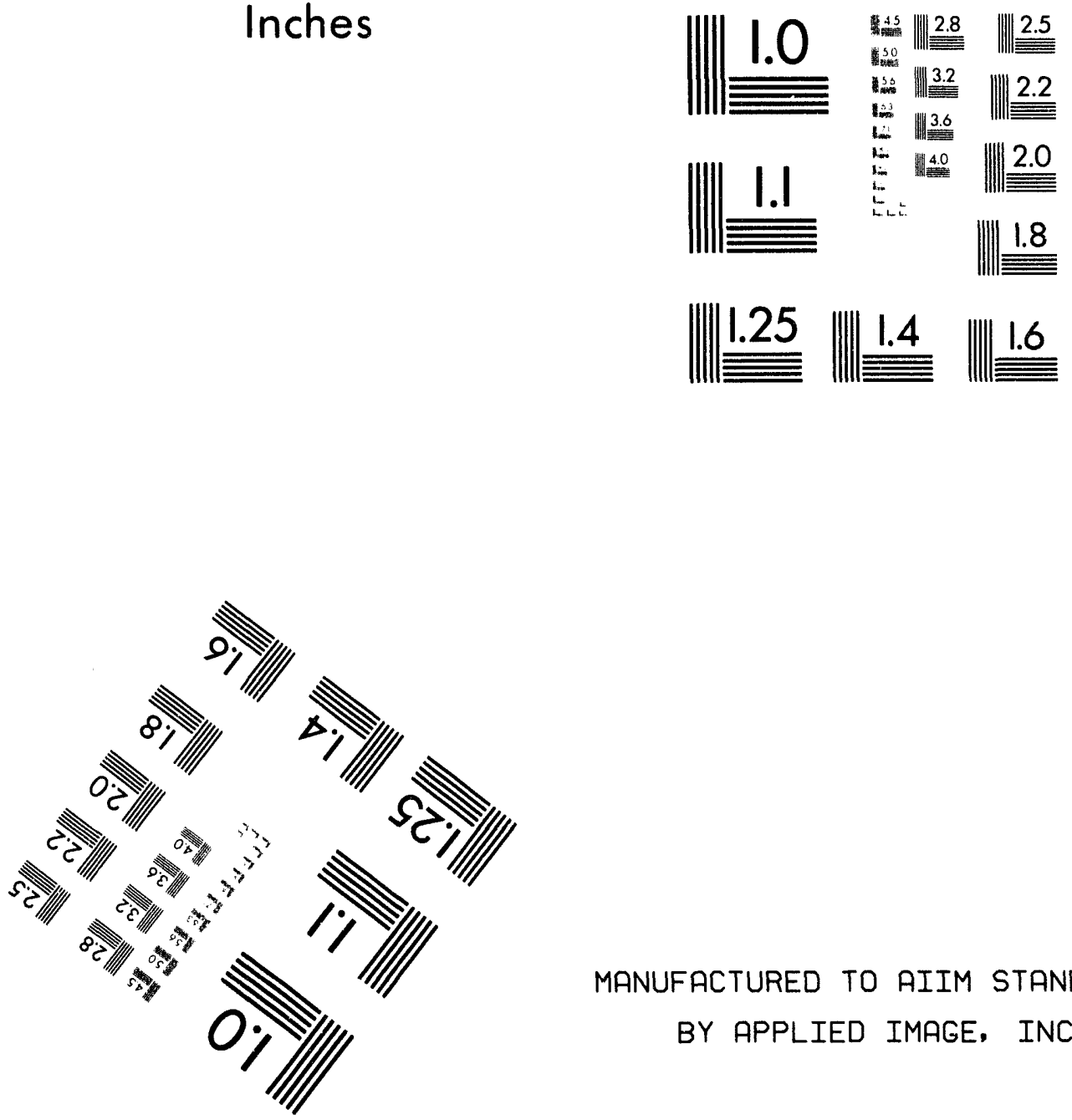

MANUFACTURED TO AIIM STANDARDS BY APPLIED IMAGE, INC.

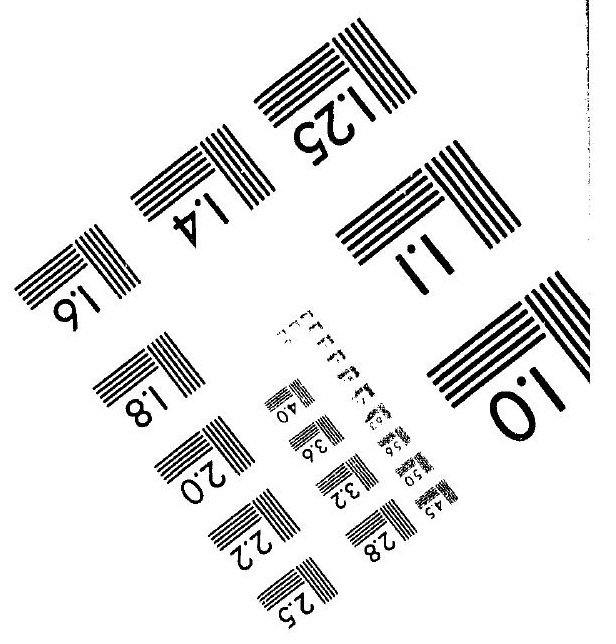



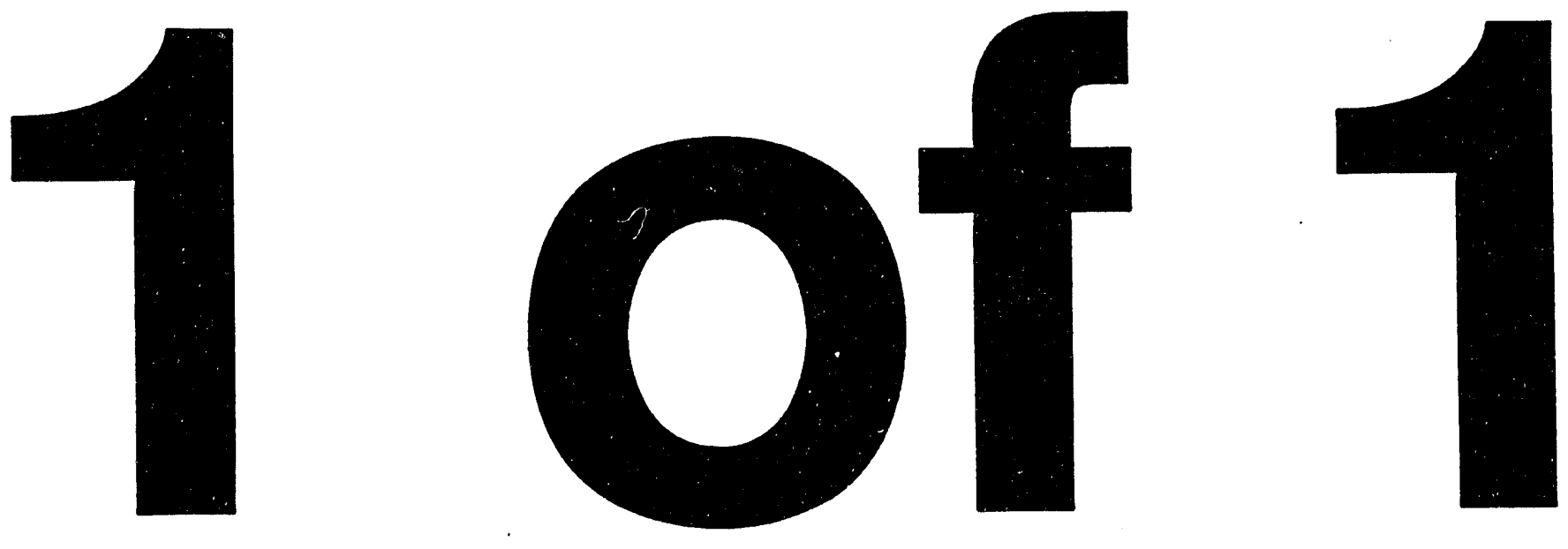


\section{GAMQUEST, a Computer Program to Identify Gamma Rays}

\section{Edgardo Browne}

Nuclear Science Division

Lawrence Berkeley Laboratory

University of California

Berkeley, California 94720

USA

\section{May 1994}

This paper describes the application of the computer program GAMQUEST to the study of gamma-ray spectra. The program is especially suited to the analysis of samples produced by neutron activation, and of environmental samples containing radioactive pollutants. GAMQUEST searches a large database (with data for over 60,000 gamma rays) to identify the various spectral lines from samples. The program runs on the VAX/6610 computer cluster of the Lawrence Berkeley Laboratory, and can be accessed from individual accounts or through Hepnet, Internet, or World Wide Web networks.

This work was supported by the Director, Office of Energy Research, Office of High Energy and Nuclear Physics, Division of Nuclear Physics, of the U.S. Department of Energy under Contract DEAC03-76SF00098. 
$89^{\text {rayydes paper }}$ 


\section{Introduction}

The characteristic energies and intensities of gamma rays emitted by radioactive isotopes are commonly used as fingerprints for isotope identification. This specificity is the foundation of neutron-activation analysis and the basis for the analysis of radioactivity in environnental samples. Because of the large number (over 60,000) of known gamma rays from radioactive decay, analysis can be a formidable task, especially when applied to complex spectra with numerous gamma rays. GAMQUEST can help overcome this difficulty by comparing spectral lines with gamma-ray data contained in a large database from the Table of Radioactive Isotopes ${ }^{1}$. For each retrieved gamma ray, GAMQUEST displays the energy, intensity, the name and half-life of the emitting isotope, and the energies and intensities of the two most intense gamma rays emitted by the isotope. Additional searching conditions applied to gamma-ray intensities, and to the isotope's half-life, mass number $\mathrm{A}$, and atomic number $\mathrm{Z}$ may be included to reduce the number of superfluous matches. A second program option produces, for individual isotopes, a list of emitted $\mathrm{x}$ rays and gamma rays. The program stores the retrievals into files that may be printed, or transferred by electronic mail to other computers, as well as edited and displayed on the screen at the end of each session.

\section{Program Access and Output Files.}

GAMQUEST runs on the CSA1 and CSA2 computers of the Lawrence Berkeley Laboratory (LBL) VAX/6610 cluster of Digital Equipment Corporation (DI C) computers. The program, written in DEC Command Language (DCL) for the Virtual Memory System (VMS) operating system, executes several procedures that operate a large relational database from the Table of Radioactive Isotopes. Datatrieve ${ }^{2}$ (a DEC database management system) manages this database, which is on disk in the Common Data Dictionary (CDD). Users can run GAMQUEST from individual accounts, or from a Guest Account, through Hepnet, Internet, or World Wide Web (WWW). GAMQUEST searches the database and displays results on the screen. When running from individual accounts, it stores the retrievals on the following disk files: GAMQUEST.OUT (gamma-ray searches) and GAMLIST.OUT (lists of $x$ rays and gamma rays). These files remain a /ailable on disk at the end of sessions. GAMQUEST, however, deletes them at the beginning of a new session. This version of the program is available from the LOCAL_TOOLS public domain of the LBL computer cluster. When running GAMQUEST from a Guest Account, users can display the retrievals on the screen and transfer them (by electronic mail) to other disks or computers, but cannot print results. At the end of each session, the program deletes the files that contain the retrievals.

\section{How to Run GAMOUEST.}


From individual accounts:

a. Enter \$ LOCAL_TOOLS GAMQUEST (or store this line in your LOGIN.COM file so that it will be executed every time you log into your account).

b. Enter GAMQUEST.

A guest account with the username GAMQUEST is available at the LBL VAX/6610 computer cluster. This account has limited central processing units (CPU), and does not require a password. Access to the account is possible from the Hepnet, Internet, and World Wide Web networks.

From Hepnet:

a. Enter SET HOST CSA2 (or CSA1).

b. Enter GAMQUEST in response to Username:, and proceed.

From Internet:

a. Enter TELNET CSA2 .LBL.GOV (or CSA1.LBL.GOV).

b. Enter GAMQUEST in response to Username: , and proceed.

From World Wide Web:

a. Display LBL's World Wide Web home page (http://www.lbl.gov/LBL.html).

b. Look under the Scientific Programs link, and proceed. 


\section{Gamma-Ray Data.}

Gamma-ray energies and intensities displayed by GAMQUEST are rounded values, without uncertainties, from the Table of Radioactive Isotopes. This publication has detailed specific information on these quantities.

The energy of a gamma ray observed in the decay of different isotopes, but from the de-excitation of the same level in the daughter nucleus, has one value only in the database. For example, the 1460.8-keV gamma ray which de-excites the $1460.8-\mathrm{keV}$ level in ${ }^{40} \mathrm{Ar}$, has the same energy in both the $\beta^{-}$decay of ${ }^{40} \mathrm{Cl}$ and in the electron-capture decay of ${ }^{40} \mathrm{~K}$.

Gamma-ray intensities are given either on an absolute scale (\%), per 100 disintegrations of the emitting isotope, or on a relative scale ( $r$ ), usually normalized to 100 for the most intense gamma ray. These intensities may be compared with those deduced from the gamma-ray spectra.

Gamma rays that de-excite an isomeric state in the daughter nucleus have equilibrium intensities in the database. These values are reached after about three half-lives of the isomeric state. For example, the $140.5-\mathrm{keV}$ gamma ray from the decay of ${ }^{99} \mathrm{Mo}(2.7 \mathrm{~d})$ has an intensity of $90.7 \%$ in equilibrium with the daughter nucleus ${ }^{99} \mathrm{Tc}(6.0 \mathrm{~h})$.

$\mathrm{X}$-ray intensities, which are always given on an absolute scale (\%), have been calculated from the number of vacancies in the $K, L_{1}, L_{2}$, and $L_{3}$ atomic shells, produced both by conversion and by electron-capture processes. Unless they constitute the only source of electromagnetic radiation, $\mathrm{x}$ rays are not listed if their intensities are $<0.01 \%$ of that for the most intense gamma ray emitted by the isotope.

\section{GAMQUEST, a Tool for Applied Research}

This program has been especially tailored to the needs of applied users in gamma-ray spectroscopy, in particular, to those needs requiring the identification of gamma-ray emitters in samples. GAMQUEST assumes that users have conventional experimental capability, and optimizes the various questions and mesisages accordingly. The main menu has the following options:

- Gamma-ray Search, which identifies spectral lines by comparing their energies with database values for known isotopes. For each matched gamma ray, GAMQUEST displays (on the same line) its energy, the element symbol and half-life of the emitter, and the energies and intensities of the emitter's two most intense gamma rays. These additional data are helpful for identifying other spectral lines, 
thereby confirming their isotopic assignments. The program assumes that users analyze a spectrum to identify the various lines that, ultimately, may lead to knowledge of the emitting isotopes. In addition to the gamma-ray energy and its uncertainty, GAMQUEST allows one to search on:

Gamma-ray intensities,

Isotope's half-life range, and

Isotope's mass number $(\mathrm{A})$ and atomic number $(\mathrm{Z})$.

GAMQUEST requests an energy and its uncerta $n$ ty, followed by the number of the most intense gamma rays (of the emitting isotopes) to be included in the search; it then requests the half-life range of these isotopes. The latter information is usually obtained from the gamma-ray decay rate of the sample, or often, by comparing spectral intensities with those from known isotopes also present in the spectrum. Finally, the program requests a range for the mass number $(\mathrm{A})$ and atomic number $(\mathrm{Z})$. This information might be known from the type and origin (e.g., neutron activated, environmental, etc.) of the sample. An increase in the uncertainty of the input gamma-ray energy will augment the number of matches with gamma rays in the database, and therefore produce a lengthier gamma-ray list. However, the number of superfluous matches may be significantly reduced by including additional conditions for the half-life, mass, atomic numbers, and, especially, for the gamma-ray intensities. For example, a search for a $(122.1 \pm 0.1)-\mathrm{keV}$ gamma ray produces a list of 7 gamma rays $(7$ matches). For a $(122.1 \pm 0.5)-\mathrm{keV}$ gamma ray, the number of matches increases to 41 . This number may be reduced to 7 by including in the search just the most intense gamma ray emitted by each isotope.

- List of X Rays and Most Intense Gamma Rays, which lists energies and intensities for x rays, and for the most intense gamma rays emitted by a specific isotope. The length of the list depends on intensity conditions imposed. GAMQUEST requests a mass number, then a chemical element symbol (first letter only should be capital). If the isotope has isomers, the program displays their entries on the screen and requests users to choose one. As in the previous option (see Gamma-ray Search), GAMQUEST assumes that users analyze a spectrum to identify spectral lines, using the strongest known gamma rays from specific isotopes in the database. For each spectrum, the program requests the energy cutoff (minimum and maximum) and the number of most intense gamma rays to retrieve. GAMQUEST displays them on the screen, together with the more intense (see Section 4 ) $x$ rays emitted by this isotope.

Decay modes are used to label gamma rays from isotopes that decay through more than one mode. The isotope symbol of the daughter nucleus labels $\mathrm{x}$ rays, and the classical Siegbahn notations designate the various lines. This option not only provides the database information that is needed for isotope identification, but also may serve another purpose as well. The following example illustrates this point. 
Suppose a user enters $1332.3 \pm 0.1 \mathrm{keV}$, expecting to find ${ }^{60} \mathrm{Co}$, but ${ }^{6(} \mathrm{Co}$ does not appear on the retrieval list. Inspection of the full list of ${ }^{60} \mathrm{Co}$ photons shows an energy of $1332.5 \mathrm{keV}$, which is outside the input energy range, and obviously the reason for its absence from the retrieval.

\section{Searching Strategies.}

Efficient searches should minimize the number of superfluous matches without missing useful data. Although each case may be different, the following general suggestions should help accomplish this objective.

\section{- Input gamma-ray energies and uncertainties.}

To avoid missing useful matches (even when using precise energies) it is recommended that uncertainties be increased by a factor of at least three, or more, if there are indications of systematic uncertainties.

\section{- Gamma-ray intensities.}

Prominent lines in the spectrum should be identified first, using only the few most intense (e.g., 3 to 5) gamma rays from each isotope in the database.

\section{- Half-life, atomic (Z), and mass (A) numbers.}

Additional conditions on the range of these properties would further reduce the number of superfluous matches. For example, if the sample was produced by neutron activation, and the gamma-ray measurement started a week after bombardment, it is very likely that most of the gamma rays in the spectrum belong to isotopes with half-lives greater than about one day. A range of 1 day to $1.0 \times 10^{9} \mathrm{y}$ would be adequate for the search. There may be samples for which the ranges of $\mathrm{A}$ and $\mathrm{Z}$ are known (by chemical or isotopic separation, etc.). These ranges should be included as conditions to the search.

\section{- X-ray and gamma-ray lists.}

Full lists of gamma and $\mathrm{x}$ rays should be retrieved for the gamma-ray emitters assigned in the previous steps. Additional lines may be identified here, confirming those assignments.

\section{- Other conditions.}

If weaker lines remain unidentified in the spectrum, new searches should be performed, with less restrictive conditions. These searches will obviously produce lengthier lists of gamma rays and an increased number of superfluous matches. 


\section{Examples.}

\section{- Neutron Activation Analysis.}

A rock sample from the Sudbury Neutrino Observatory (SNO) in Canada was bombarded with thermal neutrons in a reactor for one hour, and then analyzed for content. Figure 1 shows a gamma-ray spectrum measured for 30 minutes, 10 days after the end of bombardment. The following conditions should be adequate for the first search with GAMQUEST:

a. Uncertainty in gamma-ray energies: $1 \mathrm{keV}$.

b. Number of most intense gamma rays from each isotope: 5 .

c. Half-life range: 1 day to $1 \times 10^{9} \mathrm{y}$.

d. No conditions on atomic or mass numbers.

To run the program from an individual account, type GAMQUEST and proceed (See Section 3). Entries in boldface are user's responses.

The program displays the following message:

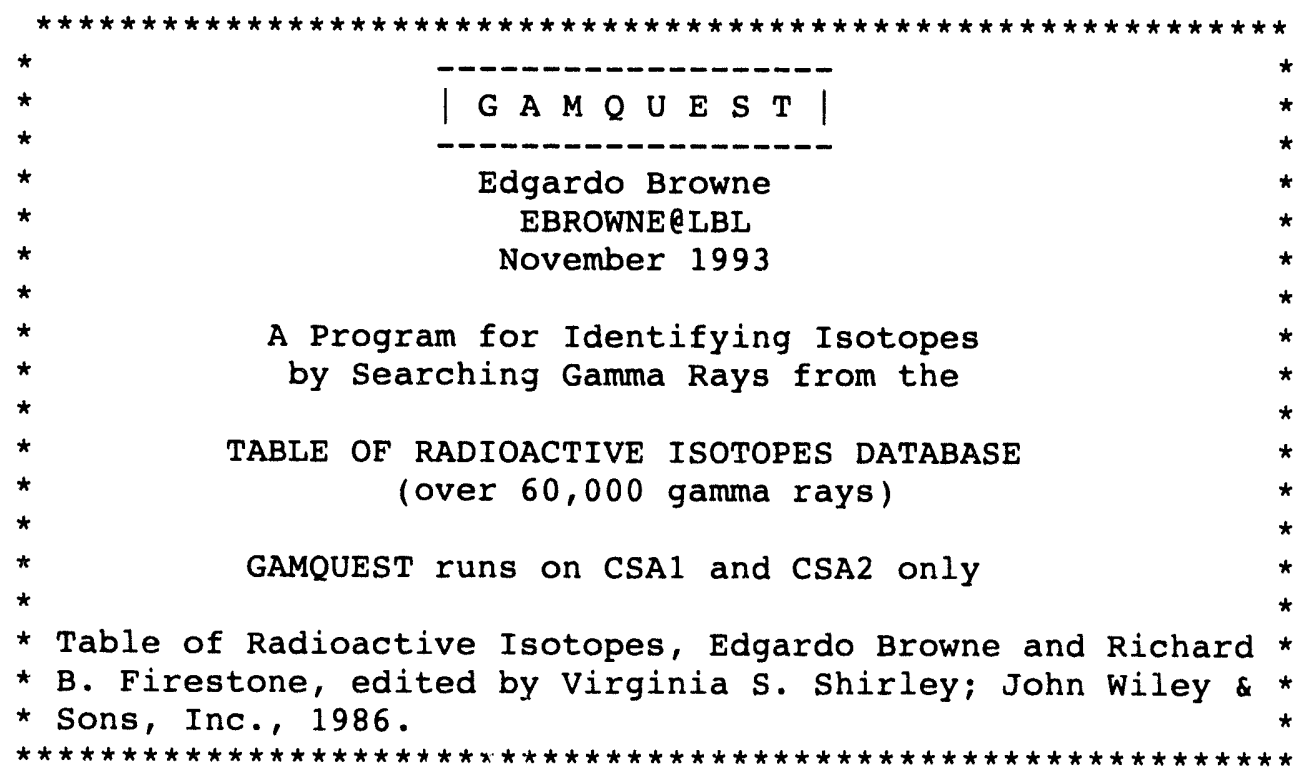

Press RETURN to continue 
Then GAMQUEST displays its main menu:

Enter:

1. To perform a gamma-ray search.

2. To list gamma and $x$ rays by emitting isotope.

3. TO QUIT.

Enter 1, 2, or 3: 1

This section of the program presents gamma rays and their emitting isotopes. GAMQUEST lists gamma rays from the Table of Radioactive Isotopes (and their emitting isotopes) for energies within a specified input range (Eg +- DEg).

In addition, for each listed isotope, the program displays the two most intense gamma rays. Intensities are:

* Photon emission probabiljties ( 8 ) (per 100 disintegrations of the emitting isotope), or

* Relative $(r)$, usually normalized to 100 for the most intense gamma ray.

Additional searching conditions are:

* Number of most intense gamma rays from each isotope.

* Half-life, and/or

* Mass number (A), and/or

* Atomic number ( $Z$ ).

The entire retrieval is stored in the file GAMQUEST.OUT, which may be displayed, edited, or printed at the end of the session. Searches may be repeated using the same or different input data. Please allow 30 seconds to load the database.

L O A D I N G D A T A B A S E ...

Enter gamma-ray energy (keV), (-1 for NEW retrieval, 0 to QUIT search): 103.4 Enter Uncertainty (keV): 1

Enter number of most intense gamma rays (e.g., $1,4,5, \ldots),(0$ to CANCEL $): 5$ Do you want to set a range for the isotope's half-life?

Enter $\mathrm{Y}$ or $\mathrm{N}$ : $\mathrm{Y}$

These are the half-life units:

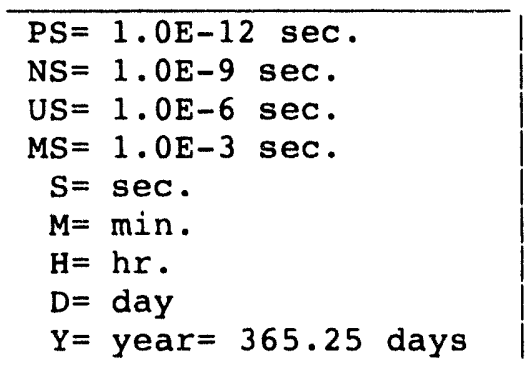


Enter minimum half-life (e.g., 20.0, 3.1E10, etc.). No unit: 1

Enter unit (e.g., S, M, H, D, Y, etc.), (C to CANCEL): d

Enter maximum half-life (e.g., 20.0, 3.1E10, etc.). No unit: 1E9

Enter unit (e.g., S, M, H, D, Y, etc.), (C to CANCEL): Y

Do you want to set a range for the isotope's atomic number $Z$ ?

Enter $\mathrm{Y}$ or $\mathrm{N}$ : $\mathbf{n}$

**NO RESTRICTION ON 2 **

Do you want to set a range for the isotope's mass number $A$ ?

Enter $\mathbf{Y}$ or $\mathbf{N}$ : $\mathbf{N}$

**NO RESTRICTION ON A**

\begin{tabular}{|c|c|c|c|c|c|c|c|c|c|c|c|c|c|}
\hline v) & I & & & sotope & (T1/ & & & ev) & $\begin{array}{c}\text { Two mo } \\
\text { I }\end{array}$ & & $n$ & $\begin{array}{l}\text { ise gamma } \\
\mathrm{E} \text { (keV) }\end{array}$ & $\begin{array}{l}\text { rays } \\
\text { I }\end{array}$ \\
\hline 10 & $3.2 \mathrm{E}-$ & & 8 & $72 \mathrm{zn}$ & 1.93 & D & ] & 1.7 & ( 83.0 & & 1 & 191.5 & $93.8 \mathrm{E}-$ \\
\hline 10 & 8.3 & & 8 & $\mathrm{Sm}$ & & $D$ & ] & & 173 & 2 & 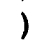 & & $E-$ \\
\hline 10 & 19.6 & & 8 & 15 & 2 & D & ] & & i 27 & & ) & 7 & $\mathrm{E}-$ \\
\hline 10 & $39.9 \mathrm{E}-$ & & 8 & & 4. & D & ] & & ( 29.1 & & 3 & .0 & $E-$ \\
\hline 50 & $78.1 \mathrm{E}-$ & 4 & 8 & 24 & 376. & $\mathrm{KY}$ & 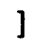 & .9 & ( $36.0 \mathrm{E}$ & 3 & ) & .81 & $E-$ \\
\hline & $10.0 \mathrm{E}-$ & & 8 & 24 & 14.4 & $Y$ & ] & .5 & ( $18.0 \mathrm{E}-$ & 5 & ) & 77.0 & E- \\
\hline & $70.0 \mathrm{E}-$ & & 8 & 24 & 6.56 & $\mathrm{KY}$ & ] & & $(45.0 \mathrm{E}-$ & 3 & ) & 160.3 & $E-$ \\
\hline & $4.6 \mathrm{E}-$ & 2 & 8 & Np & & KY & & & 127.6 & & & 45.2 & $\mathrm{OE}-$ \\
\hline
\end{tabular}

Enter gamma-ray energy (keV), (-1 for NEW retrieval, 0 to QUIT search): 192.6

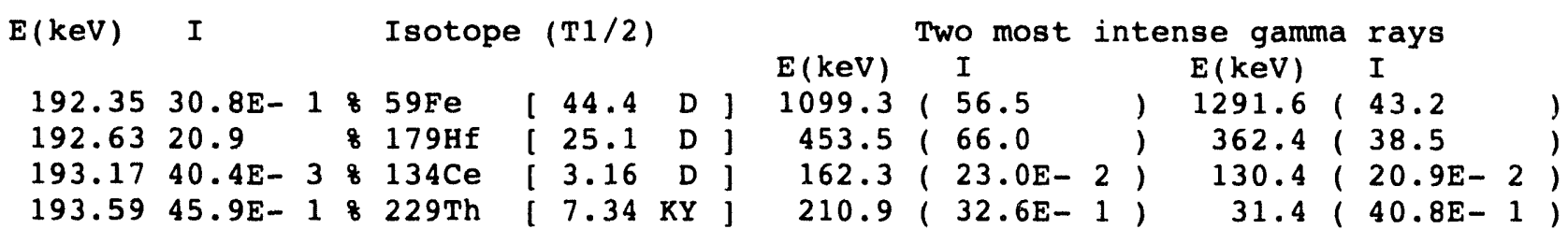

Most of the remaining gamma rays can be identified by repeating this search for each spectral line. The user can summarize the matches selected from various searches, as obtained using the same searching conditions. For this search:

\begin{tabular}{|c|c|c|c|c|c|c|c|c|c|c|c|c|}
\hline$E(\mathrm{keV})$ & I & & Isotope & $(\mathrm{T} 1 / 2)$ & & $E(k e V)$ & $\begin{array}{c}\text { Two most } \\
\text { I }\end{array}$ & & 1 & $\begin{array}{l}\text { se gamma } \\
\mathrm{E}(\mathrm{keV})\end{array}$ & $\begin{array}{l}\text { rays } \\
\text { I }\end{array}$ & \\
\hline 103.18 & 28.3 & 8 & $153 \mathrm{Sm}$ & [ 1.94 & D ] & 97.4 & ( $73.0 \mathrm{E}-$ & 2 & ) & 69.7 & $53.2 E-1$ & 1 \\
\hline 123.78 & 29.1 & 8 & $131 \mathrm{Ba}$ & {$[11.8$} & D ] & 496.3 & 143.8 & & $j$ & 216.0 & 19.9 & \\
\hline 159.38 & 68.0 & 8 & $47 \mathrm{SC}$ & {$[3.34$} & D J & & $i$ & & j & & & \\
\hline 192.35 & $30.8 \mathrm{E}-1$ & 8 & $59 \mathrm{Fe}$ & {$[44.4$} & D ] & 1099.3 & $(56.5$ & & ) & 1291.6 & 43.2 & \\
\hline 282.52 & $30.6 \mathrm{E}-1$ & 8 & $175 \mathrm{Yb}$ & {$[4.19$} & D ] & 396.3 & ( $65.0 \mathrm{E}-$ & 1 & $j$ & 113.8 & $19.1 E-1$ & 1 \\
\hline 312.01 & 36.0 & 8 & $233 \mathrm{~Pa}$ & {$[27.0$} & D j & 340.6 & ( $41.7 \mathrm{E}-$ & 1 & $j$ & 300.2 & $62.0 E-1$ & $1)$ \\
\hline 320.08 & $98.3 E-1$ & 8 & $51 \mathrm{Cr}$ & {$[27.7$} & D J & & 1 & & ) & & & \\
\hline 328.76 & 20.7 & 8 & 140La & {$[1.67$} & D ] & 1596.5 & 195.4 & & 1 & 487.0 & 45.9 & \\
\hline 396.33 & $65.0 E-1$ & 8 & $175 \mathrm{Yb}$ & [ 4.19 & D j & 113.8 & ( $1.9 .1 \mathrm{E}-$ & 1 & $j$ & 282.5 & $30.6 E-1$ & 1 \\
\hline 482.00 & 80.6 & 8 & $181 \mathrm{Hf}$ & {$[42.3$} & D j & 345.8 & 115.1 & & ) & 132.9 & 35.9 & \\
\hline 487.03 & 45.9 & 8 & $140 \mathrm{La}$ & {$[1.67$} & D j & 1596.5 & ( 95.4 & & j & 815.8 & 23.6 & \\
\hline 496.26 & 43.8 & 8 & $131 \mathrm{Ba}$ & [ 11.8 & D ] & 216.0 & ( 19.9 & & $j$ & 123.8 & 29.1 & \\
\hline 497.05 & 88.7 & 8 & $103 \mathrm{Ru}$ & [ 39.2 & D j & 557.0 & ( $83.2 \mathrm{E}-$ & 2 & ) & 610.3 & $56.4 E-1$ & 1 \\
\hline 604.71 & 97.6 & 8 & $134 \mathrm{Cs}$ & {$[2.06$} & Y $]$ & 569.3 & ( 15.4 & & ) & 795.9 & 85.4 & \\
\hline 795.87 & 85.4 & 8 & $134 \mathrm{Cs}$ & {$[2.06$} & Y J & 604.7 & ( 97.6 & & ) & 569.3 & 15.4 & \\
\hline 815.78 & 23.6 & 8 & 140La & {$[1.67$} & D $]$ & 1596.5 & 195.4 & & ) & 487.0 & 45.9 & \\
\hline & & & & & & & & & & & & \\
\hline
\end{tabular}




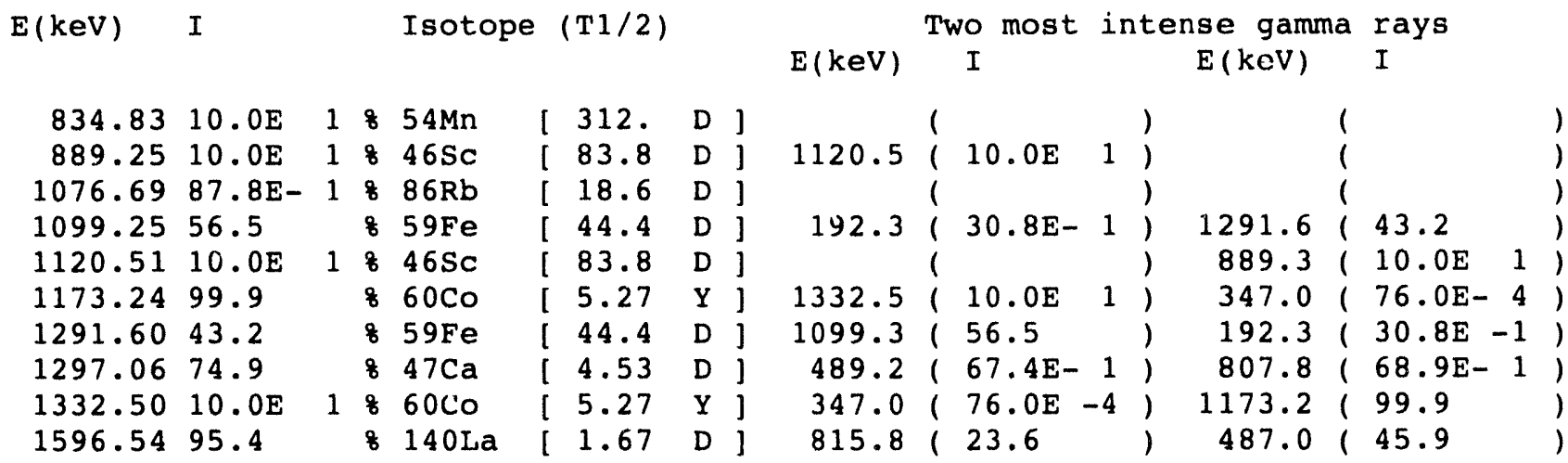

The following are selected gamma rays, which were retrieved by including all gamma rays in the search, irrespective of intensity.

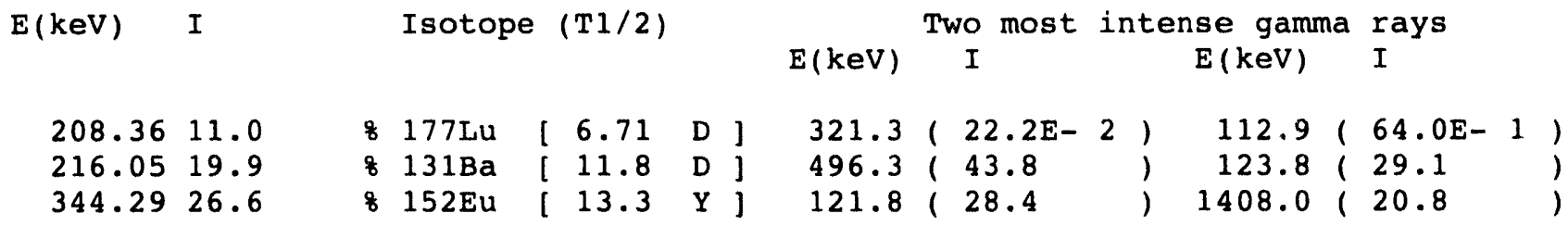

Spectral lines at $142.8,145.6,752.4,779.4,926.1,964.6$, and $1.369 .5 \mathrm{keV}$ remain unassigned, but are probably weaker gamma rays emitted by some of the isotopes listed above. Option 2 from the main menu, listing gamma-ray energies and intensities for specific isotopes, inight help to assign these gamma rays. After leaving Option 1 as follows:

Enter gamma-ray energy (keV), (-1 for NEW retrieval, 0 to QUIT search): 0

GAMQUEST.OUT contains a copy of this session (previous versions deleted).

Do you want a printout of this session? $(\mathrm{Y} / \mathrm{N}): \mathbf{N}$

GAMQUEST displays its main menu:

Enter:

1. To perform a gamma-ray search.

2. To list gamma and $x$ rays by emitting isotope.

3. TO QUIT.

Enter 1, 2, or 3: 2 
Option 2 is active now. GAMQUEST displays the following:

LIST OF GAMMA AND $X$ RAYS

This section of the program lists $x$ rays, and a number (given by user) of the most intense gamma rays emitted by a specified

isotope. GAMQUEST prompts for the isotope's mass number (A) and the element symbol (first letter should be capital). For isomers, it aiso displays half-life, and requests a choice. It then prompts for an energy range (optional), and finally, for the maximum number of gamma rays ( $x$ rays not included) to be displayed.

$\mathrm{X}-\mathrm{ray}$ intensities were deduced from atomic shell vacancies created by electron conversion and electron capture. Only reasonably precise $K$ and $L$ intensities were included in the database. Intensities are:

* Photon emission probabilities ( 8 ) (per 100 disintegrations of the emitting isotope), or

* Relative (r), usually normalized to 100 for the most intense gamma ray.

The ertire retrieval is stored in the file GAMLIST.OUT, which may be displayed, edited, or printed at the end of the session. Other listings may be created using the same or different input data. Please allow about 15 seconds to load the database. I $O$ A D I N G D A T A B A E . .

Enter parent mass number A, (-1 for NEW retrieval, 0 to QUIT): 152

Enter element symbol (e.g., Fe, Ag, etc.): Eu

152Eu has the following isomers:

$\begin{array}{ll}13.3 & \mathrm{Y} \\ 9.32 & \mathrm{H} \\ 1.60 & \mathrm{H}\end{array}$

Choose one.

Enter half-life. No units: 14

Enter half-life unit (e.g., M, D, Y, etc.): $y$

Do you want to set a photon energy range?

Enter $\mathrm{Y}$ or $\mathrm{N}$ : $\mathrm{Y}$

Enter minimum photon energy (keV): 70

Enter maximum photon energy (keV), ( 0 to CANCEL): 1600

Enter number of most intense gamma rays $(e . g ., 3,10, \ldots),(0$ to CANCEL): 10 


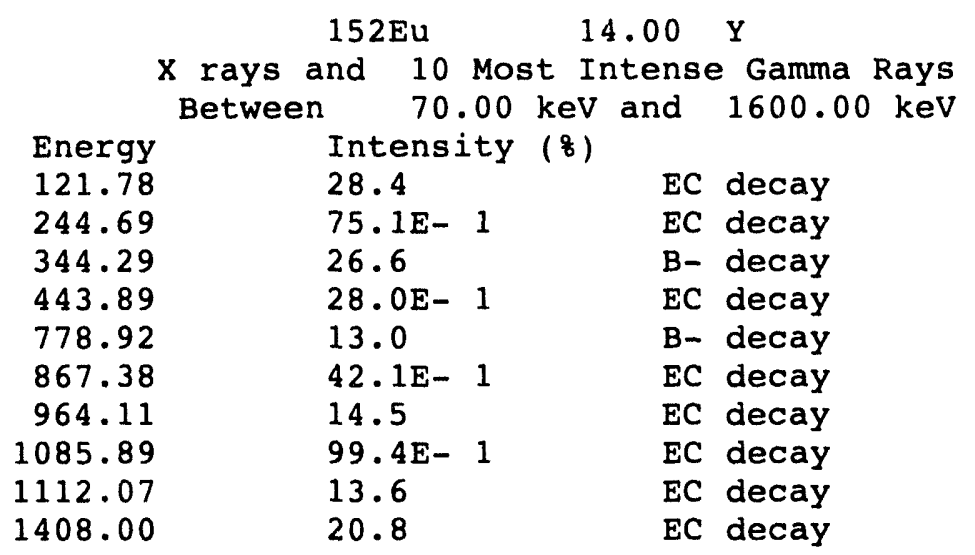

Enter parent mass number $A,(-1$ for NEW retrieval, 0 to QUIT): 0

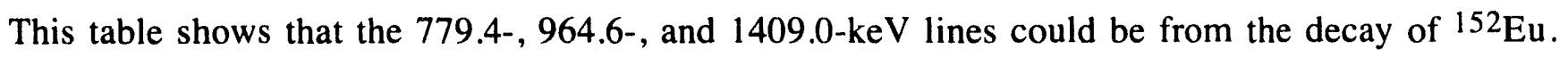
Using the same procedure, it is possible to make the following assignments: 142.8 to ${ }^{59} \mathrm{Fe}, 145.6$ to ${ }^{175} \mathrm{Yb}, 752.4$ and 926.1 (not among the ten most intense gamma rays) to ${ }^{152} \mathrm{Eu}$. X-ray data are not available for ${ }^{152} \mathrm{Eu}$ in the database, and therefore not given. The only outstanding spectral line that remains unidentified is the one at $1369.6 \mathrm{keV}$. A new search using option 1 , with no conditions other than the gamma-ray energy and uncertainty, retrieves ${ }^{24} \mathrm{Na}$ as a likely candidate:

$$
1368.6010 .0 \mathrm{E} \quad 1824 \mathrm{Na}[14.6 \mathrm{H}] 3866.1(50.8 \mathrm{E}-3) 2754.0(99.9)
$$

The strong ${ }^{24} \mathrm{Na}$ activity originated from the $(\mathrm{n}, \gamma)$ reaction on ${ }^{23} \mathrm{Na}$, contained in large amounts in the rock sample.

\section{- A list of $x$ rays and gamma rays from the decay of ${ }^{192} \mathrm{Ir}(74 \mathrm{hr})$.}

After choosing option 2 from the main menu, these are the responses to GAMQUEST:

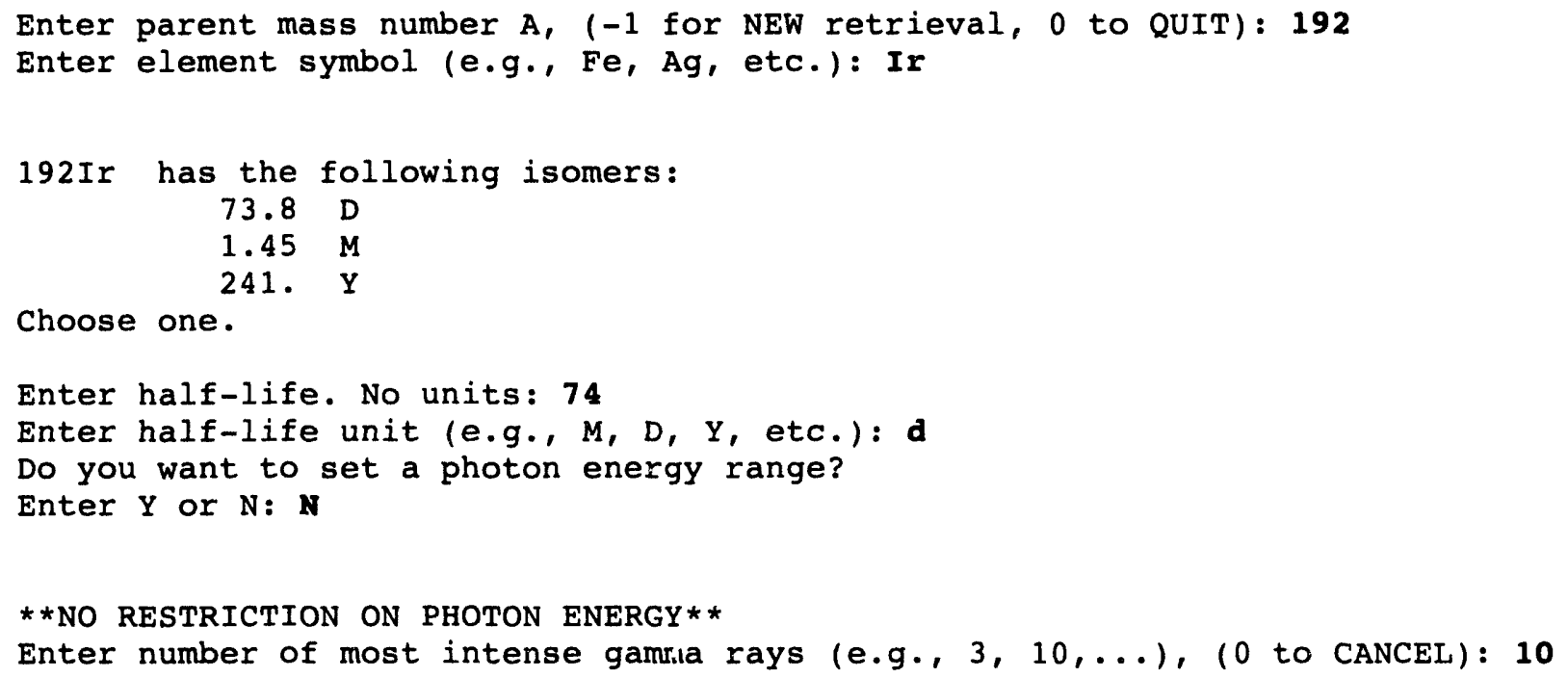




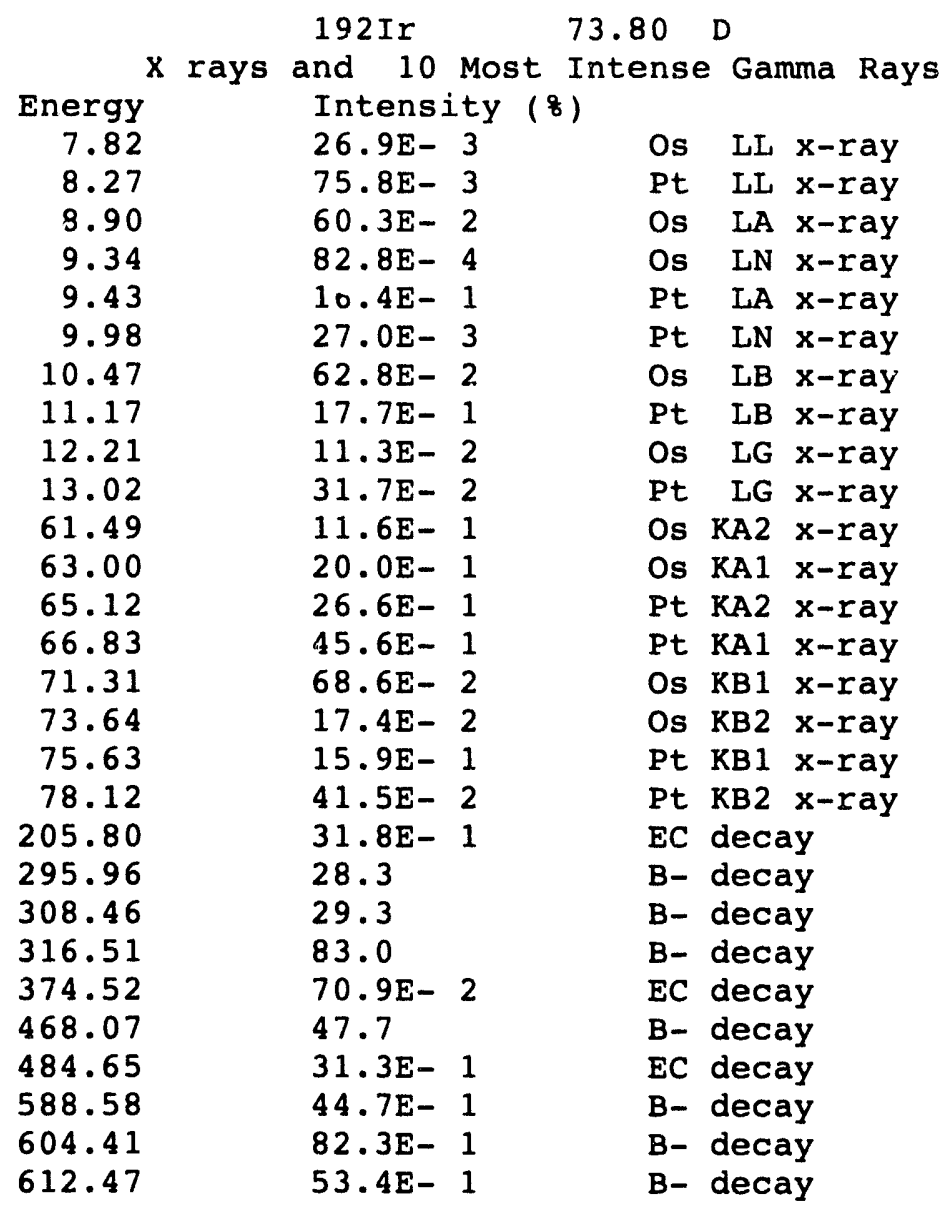

Enter parent mass number $A,(-1$ for NEW retrieval, 0 to QUIT): 0

Notice that gamma rays are labeled with the appropriate decay mode. Similarly, $x$ rays are labeled with the corresponding chemical element in which the atomic transition takes place. Intensities are given on a single scale, irrespective of which decay mode the gamma ray follows.

\section{Acknowledgments.}

I am grateful to V.S. Shirley for carefully reviewing and editing the manuscript; to R.J. Mcdonald for his invaluable suggestions which made the program more practical and easy to use; to M. Gelbaum for his assistance with the implementation of GAMQUEST into World Wide Web, and particularly, for introducing me to the enchanting new world of Mosaic; to I. Zlimen and E.B. Norman for providing their gamma-ray data on neutron activation of rock samples.

This work was supported by the Director, Office of Energy Research, Office of High Energy and Nuclear Physics, Division of Nuclear Physics, of the U.S. Department of Energy under Contract DEAC03-76SF00098. 


\section{References}

1. Table of Radioactive Isotopes, Edgardo Browne and Richard B. Firestone; edited by Virginia S. Shirley; John Wiley \& Sons, Inc., New York (1986).

2. Datatrieve, a database management system from Digital Equipment Corporation, Maynard, Massachusetts. 


\section{Figure Captions}

Figure 1. Gamma-ray spectrum of a rock sample activated by neutrons (Source: the Sudbury Neutrino Observatory (SNO)). 

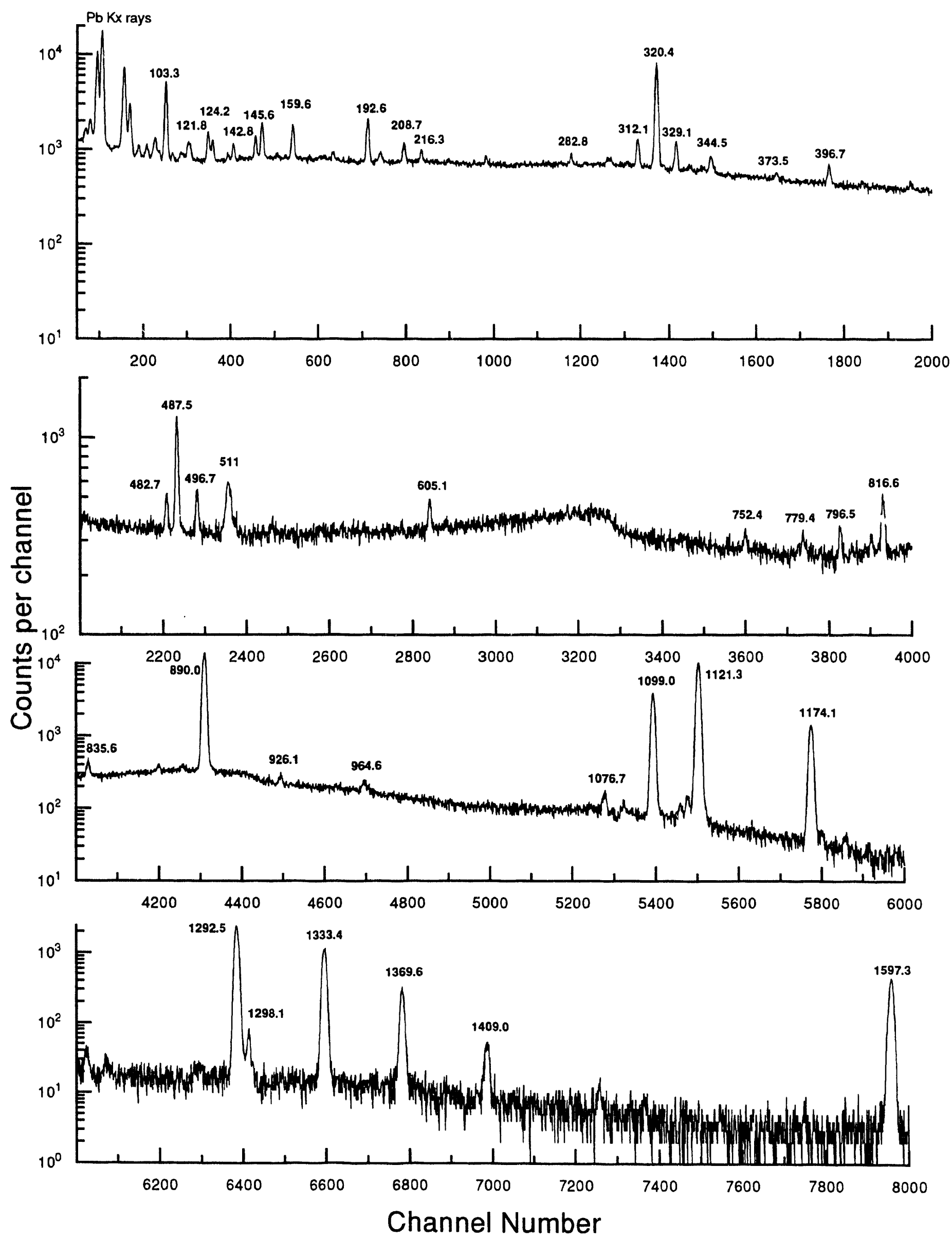

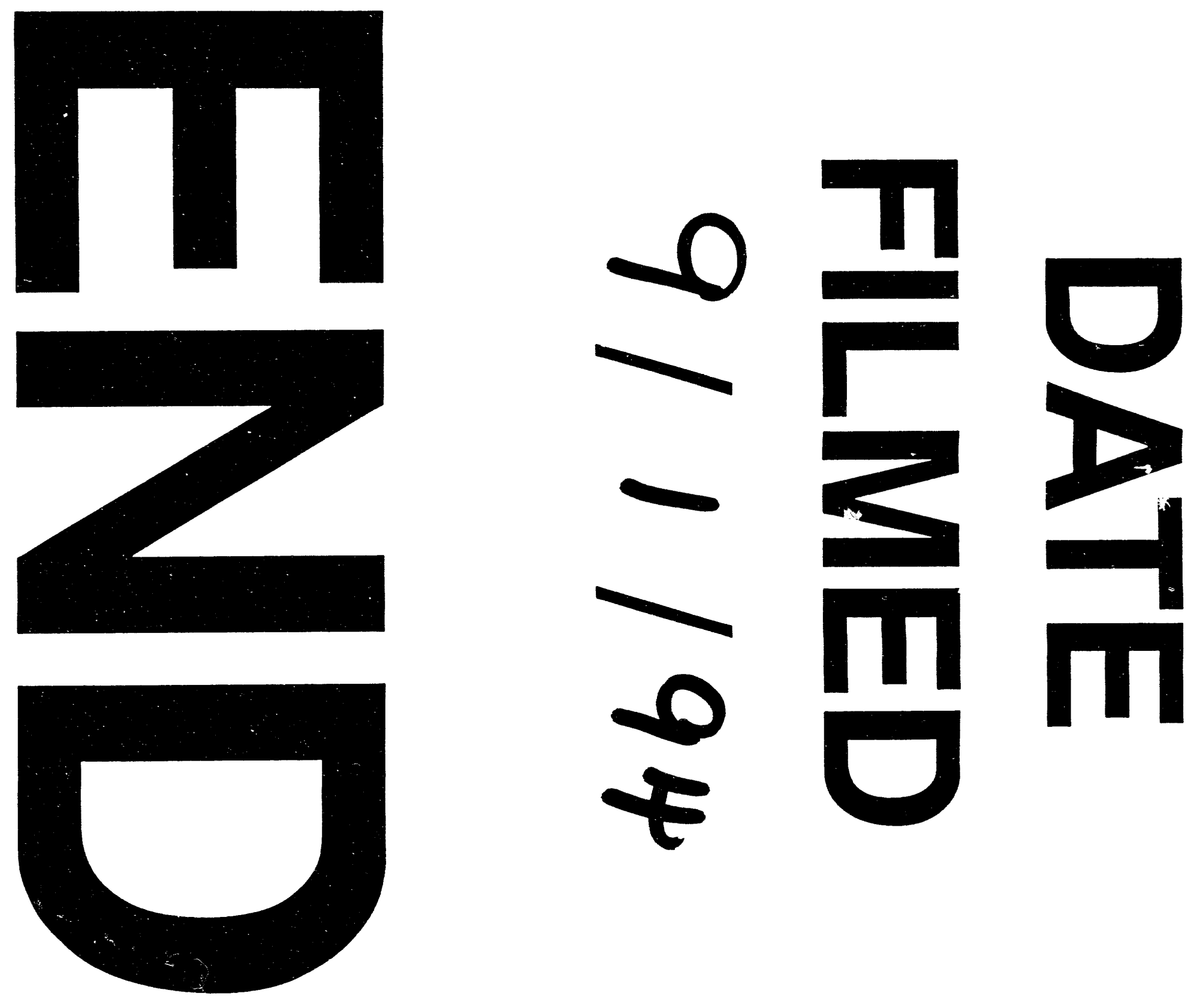
